\title{
Classical-quantum correspondence for two-level pseudo-Hermitian systems
}

\author{
K. Raimundo,, , M. C. Baldiotti,, , $\mathrm{H}$ R. Fresneda,, , 团 and C. Molina ${ }^{3, \text { S }}$ \\ ${ }^{1}$ Departamento de Física, Universidade Estadual de Londrina, 86051-990, Londrina-PR, Brazil. \\ ${ }^{2}$ Universidade Federal do ABC, Av. dos Estados 5001, 09210-580, Santo André-SP, Brazil. \\ ${ }^{3}$ Escola de Artes, Ciências e Humanidades, Universidade de São Paulo, \\ Av. Arlindo Bettio 1000, CEP 03828-000, São Paulo-SP, Brazil.
}

\begin{abstract}
In this work, a classical-quantum correspondence for two-level pseudo-Hermitian systems is proposed and analyzed. We show that the presence of a complex external field can be described by a pseudo-Hermitian Hamiltonian if there is a suitable canonical transformation that links it to a real field. We construct a covariant quantization scheme which maps canonically related pseudoclassical theories to unitarily equivalent quantum realizations, such that there is a unique metric-inducing isometry between the distinct Hilbert spaces. In this setting, the pseudo-Hermiticity condition for the operators induces an involution which guarantees the reality of the corresponding symbols, even for the complex field case. We assign a physical meaning for the dynamics in the presence of a complex field by constructing a classical correspondence. As an application of our theoretical framework, we propose a damped version of the Rabi problem and determine the configuration of the parameters of the setup for which damping is completely suppressed. The experimental viability of the proposal is studied within a specific context. We suggest that the main theoretical results developed in the present work could be experimentally verified.
\end{abstract}

\section{INTRODUCTION}

The simplest system with nontrivial dynamics that we can build in quantum mechanics is the two-level system. But despite its simplicity, one cannot underestimate the power of this setup. For instance, two-level models are the best understood quantum systems and adequately describe several physically relevant scenarios. Moreover, they play an important role in the understanding of more intricate arrangements. In general, one can treat a quantum two-level system as a spin- $1 / 2$ particle interacting with an external magnetic field if the spatial dynamics is not taken into account. Thus, a two-level system is governed by the Pauli equation in $(0+1)$ dimension,

$$
\mathrm{i} \frac{\partial v}{\partial t}=\hat{H} v, \text { with } \hat{H}=\frac{\boldsymbol{\sigma}}{2} \cdot \mathbf{F} \text { and } v=\left(\begin{array}{c}
v_{1}(t) \\
v_{2}(t)
\end{array}\right) .
$$

In Eq. (1), $v$ is a two-component spinor, $\boldsymbol{\sigma}=\left(\sigma_{1}, \sigma_{2}, \sigma_{3}\right)$ are the Pauli matrices and $\mathbf{F}=\left(F_{1}(t), F_{2}(t), F_{3}(t)\right)$ represents an external field 1 Therefore, solving a two-level system is equivalent to solving (11), to which will be referred as the spin equation (SE).

Among the exact solutions of the SE, we highlight the Rabi problem [1, 2], which has applications in a wide variety of fields, such as quantum optics, quantum computing, condensed matter, molecular, atomic and particle physics. Two-level systems can also be used as a model for open systems, those which interact with the environment in which they are embedded. Although the interaction problem is well-formulated in classical physics, it is not yet fully comprehended at the quantum level. One of the reasons for the lack of a proper quantum description of the interacting process is that open systems are often described by non-Hermitian Hamiltonians [3], and consequently, by nonunitary theories. Due to the probabilistic interpretation of quantum mechanics, the notion of a nonunitary theory raises important questions. Despite that, nonunitary theories have drawn some attention in the physics community through the study of a certain class of non-Hermitian operators called pseudo-Hermitian operators (PHOs). PHOs define the so-called pseudo-Hermitian quantum mechanics (PHQM). In PHQM, the freedom in defining an inner-product in the physical Hilbert spaces is explored to recover unitarity. Therefore, one may think that the notion of nonunitarity arises because one is using the "wrong" inner product.

The freedom in choosing the inner product has already been studied [4 9]. These early developments attempted to recover unitarity from systems using what they called indefinite-metrics quantum theories (the terminology "indefinitemetrics" stands for nonpositive-definite inner products). More recently, non-Hermitian Hamiltonians with real eigen-

\footnotetext{
* kesley@uel.br

$\dagger$ baldiotti@uel.br

$\ddagger$ rodrigo.fresneda@ufabc.edu.br

$\S$ cmolina@usp.br

${ }^{1}$ We are setting $\gamma=-1$, where $\gamma=g q / 2 m$ with $q, m$ and $g$ being, respectively, the charge, mass and the $g$-factor of the spin- $1 / 2$ particle. Also, in this description, $\mathbf{F}$ has dimension of energy.
} 
values were considered (see, for example, Ref. [10]). Later on, a series of papers [11 15] exploring whether a Hamiltonian $\hat{H}$ must be Hermitian or not were proposed. The authors argued that a weaker and physically transparent condition for the reality of the spectrum of $\hat{H}$ is the presence of $\mathcal{P} \mathcal{T}$ symmetry, where $\mathcal{P}$ stands for the parity operator and $\mathcal{T}$ stands for the time-reversal operator 2 Also, it was shown that if $\hat{H}$ has an unbroken $\mathcal{P} \mathcal{T}$ symmetry, there is an operator $\mathcal{C}$, commuting with $\hat{H}$, that allows one to define a positive-definite inner product, with a metric operator given by $\eta=\mathcal{C P} \mathcal{T}$.

The issue of what are the necessary and sufficient conditions for the reality of the spectrum of a linear operator were explored in Refs. [16 20]. It turns out that the answer to this problem propelled the research in PHQM. It was shown that, albeit relevant, the role played by the $\mathcal{P} \mathcal{T}$ symmetry and the $\mathcal{C}$ operator is not a fundamental one. Indeed, it can be seen from PHQM that $\eta=\mathcal{C P} \mathcal{T}$ is just an example of a positive-definite metric operator 21]. In fact, the existence of a preferred metric, and its physical meaning, is an open issue in the PHQM. There are several contexts where pseudo-Hermitian operators appear 21]. In special, recent treatments of topological aspects of non-Hermitian systems use the framework of PHQM [22 31].

A subtle point regarding quantization in general, and quantization in the PHQM framework in particular, is that canonical transformations, which are transformations on the level of the algebra of operators, do not necessarily translate as isometries or unitary transformations between the Hilbert spaces upon which these operators act [32]. When one is faced with nonunitary canonical transformations, for instance, in the infinite-dimensional case, a physical meaning for these transformations can be established by looking at the classical limit of the theory [19, 21, 33]. This procedure is called $\eta$-pseudo-Hermitian canonical quantization.

For the present work, the important observation is that there is no usual classical analog for a system with fermionic degrees of freedom. Nevertheless, quantization schemes can still be defined in the context of pseudoclassical mechanics 34 36], in which Grassmann variables are used as phase-space coordinates. In this picture, the Grassmanian degrees of freedom should be quantized with anticommutation relations, rather than with commutation relations. The latter is of course a well-known scheme for quantization of fermionic degrees of freedom, such as spin.

In this paper, the pseudo-Hermitian treatment will be extended to the pseudoclassical framework. Despite the existing treatments concerning pseudoclassical mechanics, its relation with pseudo-Hermitian theories was not yet fully analyzed. The aim of this work is to exploit the latter at the level of canonical transformations, considering both the pseudo-Hermitian quantum theory and its pseudoclassical limit. For this purpose, complex external fields, associated with nonunitary systems, will be considered. We then study the classical correspondence in order to assign a physical meaning for the complex fields. We construct a covariant quantization scheme which maps canonically related pseudoclassical theories with real and complex external fields to unitarily equivalent quantum realizations, such that there is a unique metric-inducing isometry between the distinct Hilbert spaces. In this setting, the pseudo-Hermiticity condition for the operators induces an involution which guarantees the reality of the corresponding symbols, even in the presence of complex external fields. We apply these developments to propose a damped version of the Rabi problem, which could have important implications in related areas. Furthermore, possible experimental tests for the theory are proposed.

This work is organized as follows. In Sec.[I] the basic theoretical setup is established, with a revision of the notation used in the present development. In Sec. III a classical-quantum correspondence is proposed and explored. A physical realization of the proposed theoretical framework is constructed in Sec. IV] where the Rabi problem is extended and its generalization analyzed. Also in this section, we propose an experimental arrangement for the verification of the main theoretical results developed in the present work. In Sec. $\mathbf{V}$ final remarks and future perspectives are presented. Further details on the pseudoclassical model considered and the quantization procedure employed are presented in Appendixes $\mathrm{A}$ and $\mathrm{B}$. Units where $\hbar=1$ are used in this work, except where otherwise indicated.

\section{PSEUDO-HERMITIAN AND PSEUDOCLASSICAL FRAMEWORKS}

\section{A. Pseudo-Hermitian theories}

Simply put, pseudo-Hermitian operators are operators which are not Hermitian or symmetric with respect to the canonical or natural inner product, but which are Hermitian with respect to some (positive-definite) inner product 3 The treatment of pseudo-Hermitian operators starts with the observation that non-Hermitian matrices (that is,

matrices that are not equal to their own conjugate transpose) can have real eigenvalues. It follows that the spectra of

\footnotetext{
${ }^{2}\langle x, \mathcal{P} \psi(t)\rangle=\psi(-x, t)$ and $\langle x, \mathcal{T} \psi(t)\rangle=\bar{\psi}(x,-t)$, where the bar denotes complex conjugation.

3 We note that there is a broader definition of pseudo-Hermiticity where the product is not necessarily positive definite 37]. This characterization takes into account operators whose eigenvalues appear as complex-conjugate pairs. The restriction to the real spectrum is sometimes referred to as cryptoHermitian or quasi-Hermitian [38].
} 
the related operators can be associated with physical observables in the quantum description of a system. Taking a pseudo-Hermitian operator as the Hamiltonian of the system, an evolution operator can be constructed in such way that the time evolution is unitary [21]. This formalism is the base of the pseudo-Hermitian quantum mechanics.

Pseudo-Hermitian operators in general will not have orthogonal eigenvectors corresponding to distinct eigenvalues, as do Hermitian and normal operators. Despite of this problem, the familiar probabilistic interpretation of quantum mechanics can be recovered with a convenient choice of inner product.

Let us consider the pseudo-Hermitian formalism associated with the problem at hand. Let $\mathcal{H}$ be a finite-dimensional Hilbert space isomorphic to $\mathbb{C}^{n}$ with the canonicat 4 inner product $\langle\cdot, \cdot\rangle, \mathcal{H} \simeq\left(\mathbb{C}^{n},\langle\cdot, \cdot\rangle\right)$. We denote the adjoint of an operator $T$ with respect to the canonical inner product to be $T^{\dagger}$.

Now let $\eta: \mathcal{H} \rightarrow \mathcal{H}$ and define

$$
\langle x, y\rangle_{\eta} \equiv\langle x, \eta y\rangle, \forall x, y \in \mathbb{C}^{n} .
$$

The sesquilinear form $\langle\cdot, \cdot\rangle_{\eta}$ is an inner product in $\mathbb{C}^{n}$ if and only if

$$
\eta=P^{\dagger} P
$$

for some invertible $P$. Let us denote this new Hilbert space as $\mathcal{H}_{\eta} \simeq\left(\mathbb{C}^{n},\langle\cdot, \cdot\rangle_{\eta}\right)$. We denote $\eta$ as the metric operator. In this case, an operator $T: \mathcal{H} \rightarrow \mathcal{H}$ is pseudo-Hermitian or $\eta$-Hermitian if and only if it is symmetric with respect to the inner product (2). In other words, $T: \mathcal{H} \rightarrow \mathcal{H}$ is pseudo-Hermitian if and only if it is Hermitian as an operator on $\mathcal{H}_{\eta}$. It follows that an $\eta$-Hermitian operator $T$ satisfies

$$
T=\eta^{-1} T^{\dagger} \eta
$$

It should be noticed that the metric operator $\eta$ is not unique. In fact, if $A$ is any invertible operator which commutes with the $\eta$-Hermitian operator $T$, then $T$ is Hermitian with respect to the inner product $\langle\cdot, \cdot\rangle_{\tilde{\eta}}$ with metric $\tilde{\eta}=A^{\dagger} \eta A$.

For the specific case of the generic two-level system SE in Eq. (1), defined in terms of the Hamiltonian operator

$$
\hat{H}=\frac{1}{2}\left(\begin{array}{cc}
B_{3} & B_{1}-\mathrm{i} B_{2} \\
B_{1}+\mathrm{i} B_{2} & -B_{3}
\end{array}\right),
$$

with eigenvalues

$$
E_{ \pm}= \pm \frac{1}{2} \sqrt{B_{1}^{2}+B_{2}^{2}+B_{3}^{2}}
$$

one sees that the operator $\hat{H}$ is pseudo-Hermitian if and only if

$$
\operatorname{det}(\hat{H})=-\frac{1}{4}\left(B_{1}^{2}+B_{2}^{2}+B_{3}^{2}\right) \in \mathbb{R}_{-},
$$

since this corresponds to real eigenvalues [37].

As we will show in Sec. III A a choice of metric $\eta$ induces an isometry $\mathcal{M}$ between the Hilbert spaces $\mathcal{H}$ and $\mathcal{H}_{\eta}$ such that the Hermitian operators on $\mathcal{H}$ are mapped to Hermitian operators on $\mathcal{H}_{\eta}$. On the other hand, these operators can be seen as images of quantization maps on pseudoclassical phase spaces which are themselves related by canonical transformations. The symbols of these operators, according to each quantization map, are real functions in the respective pseudoclassical phase space.

\section{B. Pseudoclassical theories}

Let us introduce the pseudoclassical framework used in this work. Further comments on this setup are presented in Appendix A. Consider a Grassmann algebra $G_{3}(\xi)$ over the complex field $\mathbb{C}$ with generators $\xi_{i}, i=1,2,3, \xi_{i}^{2}=0$, and the pseudoclassical Lagrangian,

$$
L=\frac{\mathrm{i}}{2} \xi_{i} \dot{\xi}_{i}-H(\xi)
$$

\footnotetext{
${ }^{4}$ The canonical inner product $\langle.,$.$\rangle is defined as \langle z, w\rangle=\bar{z}_{1} w_{1}+\cdots \bar{z}_{n} w_{n}$, where $z, w \in \mathbb{C}^{n}$.
} 
By requiring that $\xi_{i}$ transform as a vector under $O(3)$, it is natural to consider a rotational- and parity-invariant theory. In this case, from the development presented in appendix $\mathrm{A}$, the Hamiltonian $H$ must be of the form

$$
H=H_{B}=-\frac{\mathrm{i}}{2} \varepsilon_{i j k} \xi_{i} \xi_{j} B_{k},
$$

where $B_{k}$ transforms as a pseudovector (for instance, like the magnetic field). Thus, the equation of motion for $\xi_{i}$ becomes

$$
\dot{\xi}_{i}=\left\{\xi_{i}, H\right\}_{D(\phi)}=-\varepsilon_{i j k} \xi_{j} B_{k}
$$

Where $\{\cdot, \cdot\}_{D(\phi)}$ is the Dirac brackets over the set of second-class constraints

$$
\phi_{i}=\pi_{i}-\frac{\mathrm{i}}{2} \xi_{i}
$$

and $\pi_{i}$ the conjugate momenta

$$
\pi_{i}=\frac{\partial L}{\partial \dot{\xi}_{i}}
$$

See Appendix A for details. We recognize (10) as the classical precession equation, like a magnetic moment immersed in a magnetic field $\mathbf{B}=\left(B_{1}, B_{2}, B_{3}\right)$.

Of particular interest for the present work is the role of involution and canonical transformations in the pseudoclassical formalism. For a general function

$$
f(\xi)=f_{0}+f_{i} \xi_{i}+f_{i j} \xi_{i} \xi_{j}+\frac{\mathrm{i}}{3 !} k_{f} \varepsilon_{i j k} \xi_{i} \xi_{j} \xi_{k},
$$

as in Eq. A3 in Appendix A we define an involution $*: G_{3}(\xi) \rightarrow G_{3}(\xi)$ such that its action on the generators $\xi_{i}$ is given by

$$
\xi_{i}^{*}=\xi_{i}, i=1,2,3
$$

Therefore, elements of the real subalgebra (those for which $f^{*}=f$ ) are given by Eq. (13) with $f_{0}, f_{i}, k_{f} \in \mathbb{R}$ and $f_{i j}=\bar{f}_{j i}$. In particular, the $*$-involution as defined above yields $H_{B}(\xi)$ in Eq. (9) to be real when $\mathbf{B} \in \mathbb{R}^{3}$. That is,

$$
\mathbf{B} \in \mathbb{R}^{3} \Longleftrightarrow H_{B}(\xi)=H_{B}^{*}(\xi)
$$

Suppose we consider a linear canonical transformation on the pseudomechanical phase space, defined as a map $(\xi, \pi) \mapsto(\zeta, \varpi)$, which preserves the symplectic structure in that the only nonvanishing Poisson brackets between the new coordinates are $\left\{\zeta_{i}(\xi, \pi), \varpi_{j}(\xi, \pi)\right\}=\delta_{i j}$. Due to the constraints $\phi$ in Eq. (11), we observe that $\pi$ is proportional to $\xi$, so we write the linear canonical transformation simply as

$$
\zeta_{i}=R_{i k} \xi_{k} \text { and } \varpi_{j}=R_{j l} \pi_{l}
$$

Then, demanding that this transformation is canonical implies $R R^{T}=\mathbb{I}$, that is, $R$ is an orthogonal matrix. In principle, $R$ can have complex entries, so $R \in O(3, \mathbb{C})$. Furthermore, under this transformation the Hamiltonian function (9) becomes

$$
H_{F}(\zeta)=-\frac{\mathrm{i}}{2} \varepsilon_{i j k} \zeta_{i} \zeta_{j} F_{k}
$$

where

$$
F_{k}=(\operatorname{det} R) R_{k l} B_{l}
$$

Relation (18) implies that

$$
F^{2}=F_{i} F_{i}=(\operatorname{det} R)^{2} R_{i j} R_{i k} B_{j} B_{k}=\delta_{j k} B_{j} B_{k}=B^{2}
$$

Thus, if $\mathbf{B}$ is a real field, then from the previous relation it follows that $F^{2}$ is a positive real number for an arbitrary complex field $\mathbf{F}$. 
Indeed, considering a complex field $\mathbf{F}$, one can define an involution such that (17) is real with respect to the new involution. Initially, let us look at functions on the Grassmann algebra $G_{3}(\zeta)$ with generators $\left\{\zeta_{i}\right\}_{i=1}^{3}$, which are given by

$$
g=g^{0}+g_{i}^{1} \zeta_{i}+g_{i j}^{2} \zeta_{i} \zeta_{j}+\mathrm{i} k_{g} \frac{1}{3 !} \varepsilon_{i j k} \zeta_{i} \zeta_{j} \zeta_{k}
$$

Then an involution $+: G_{3}(\zeta) \rightarrow G_{3}(\zeta)$ can be defined, whose action on generators is given by

$$
\zeta^{+}=\zeta^{*}
$$

where the $*$-involution is presented in Eq. (14) and the $\zeta$-terms above are taken as function of $\xi$. As a result, the even subalgebra of $G_{3}(\zeta)$ is given by the functions (20) with $g^{0} \in \mathbb{R}, g^{1}=R R^{\dagger} \bar{g}^{1}, R^{T} g^{2} R=\left(R^{T} g^{2} R\right)^{\dagger}$, and $k_{g} \in \mathbb{R}$. One can also show that the even subalgebras of $G_{3}(\xi)$ and $G_{3}(\zeta)$ are isomorphic, since $f=f^{*} \Leftrightarrow g=g^{+}$where $f(\xi)=g(\zeta(\xi))$. It follows that the Hamiltonian function $H_{F}(\zeta)$ in Eq. (17) is real with respect to the + involution (21), that is,

$$
\mathbf{B} \in \mathbb{R}^{3} \Longleftrightarrow H_{F}(\zeta)=H_{F}^{+}(\zeta) \text {. }
$$

\section{CLASSICAL-QUANTUM CORRESPONDENCE}

\section{A. Quantization and hermiticity}

In this section we develop the quantization procedure employed in the present work. Complementary material is presented in Appendix B. Analogously to the classical case (16), let us consider the canonical transformation $\zeta_{i}=R_{i j} \xi_{j}$ with $R \in O(3, \mathbb{C})$. As it is described in detail in Appendix B. on the Grassmann algebras $G_{3}(\xi)$ and $G_{3}(\zeta)$ we can define quantization maps $Q: G_{3}(\xi) \rightarrow L(\mathcal{H})$ and $Q^{\prime}: G_{3}(\zeta) \rightarrow L\left(\mathcal{H}_{\eta}\right)$. A natural question is then what is the relation between $Q(f)$ and $Q^{\prime}(g)$, where $g(\zeta)=f(\xi(\zeta))$. To address this issue, let us take $P=\mathcal{M}^{-1}$ in the expression (3), $\eta=P^{\dagger} P$, so

$$
\eta=\left(\mathcal{M} \mathcal{M}^{\dagger}\right)^{-1}
$$

Then, from Eq. (2), we see that $\mathcal{M}: \mathcal{H} \rightarrow \mathcal{H}_{\eta}$ is the isometry

$$
\langle\phi, \psi\rangle=\langle\mathcal{M} \phi, \mathcal{M} \psi\rangle_{\eta}
$$

for all $\phi, \psi \in \mathbb{C}^{2}$. Thus, for $\phi^{\prime}=\mathcal{M} \phi$ and $\psi^{\prime}=\mathcal{M} \psi$, one has

$$
\left\langle\phi^{\prime}, Q^{\prime}(g) \psi^{\prime}\right\rangle_{\eta}=\left\langle\phi, \mathcal{M}^{-1} Q^{\prime}(g) \mathcal{M} \psi\right\rangle
$$

Since $f$ and $g$ represent the same classical state (i.e., are related by a canonical transformation), one has the familiar relation between the operators of the corresponding functions:

$$
Q^{\prime}(g)=\mathcal{M} Q(f) \mathcal{M}^{-1}
$$

Moreover, let $Q^{\prime+}(g)$ denote the adjoint of $Q^{\prime}(g)$ in the inner product $\langle\cdot, \cdot\rangle_{\eta}$ in Eq. (2). It follows from the definition (2) that $Q^{\prime+}(g)=\eta^{-1} Q^{\prime \dagger}(g) \eta$. Using the results (24) and (26), it is obtained that $Q^{\prime+}(g)=\mathcal{M}^{\dagger}(f) \mathcal{M}^{-1}$. Thus, for real $g$ [with respect to the + involution presented in Eq. (21)], the corresponding operator is symmetric, $Q^{\prime+}(g)=Q^{\prime}(g)$, since real $g\left(g^{+}=g\right)$ implies real $f\left(f=f^{*}\right)$, and $Q^{\dagger}(f)=Q(f)$. The similarity relation (26) preserves the canonical relation [see relation (B6) in Appendix B],

$$
\left[Q\left(\xi_{i}\right), Q\left(\xi_{j}\right)\right]=\delta_{i j}
$$

and can be regarded as a quantum canonical transformation induced by the classical canonical transformation (16).

By means of the relation $\eta=\left(\mathcal{M} \mathcal{M}^{\dagger}\right)^{-1}$, we see that $\eta \rightarrow \eta$ if $\mathcal{M} \rightarrow \mathcal{M U}$, for unitary $U, U^{\dagger}=U^{-1}$. Let us call $Q_{U}^{\prime}$ the quantization map with isometry $\mathcal{M} U$. Then, the relation between $Q^{\prime}$ in Eq. (26) and $Q_{U}^{\prime}$ is $Q_{U}^{\prime}=S^{+} Q^{\prime} S$ where $S=\mathcal{M}(\mathcal{M U})^{-1}$. That is $Q_{U}^{\prime}$ is +-unitarily equivalent 5 to $Q^{\prime}$ :

$$
\left\langle\phi, Q_{U}^{\prime} \psi\right\rangle_{\eta}=\left\langle S \phi, Q^{\prime} S \psi\right\rangle_{\eta}
$$

${ }^{5} S^{+}=\eta^{-1} S^{\dagger} \eta$ is the adjoint with respect to the $\eta$-inner product. 
and

$$
\langle S \phi, S \psi\rangle_{\eta}=\langle\phi, \psi\rangle_{\eta} .
$$

A unitary representation of the Clifford algebra (27) on $\mathbb{C}^{2}$ is given by the Pauli matrices $\sigma_{i}$ as

$$
Q\left(\xi_{i}\right)=\frac{\sigma_{i}}{\sqrt{2}} .
$$

Then, following the fermionic symmetric ordering [see relation (B1] in Appendix B], the Hamiltonian operator $\hat{H}_{B} \equiv$ $Q\left(H_{B}\right)$ [image of (9) by the quantization map $Q$ ] is

$$
\hat{H}_{B}=\frac{1}{2} \boldsymbol{\sigma} \cdot \mathbf{B} .
$$

We recognize $\hat{H}_{B}$ as the Hamiltonian for the SE (11). Given a realization of the algebra (27), it is immediate to write a realization for the operators $Q(g(\zeta))$ using relation (26). For the particular case of the Hamiltonian function $H_{F}$ in Eq. (17), one has

$$
Q^{\prime}\left(H_{F}\right) \equiv \hat{H}_{F}=\mathcal{M} \hat{H}_{B} \mathcal{M}^{-1}
$$

Since the above relation is a similarity transformation, both operators $\hat{H}_{F}$ and $\hat{H}_{B}$ have the same eigenvalues, so from this point of view $\mathcal{M}$ is a mere change of basis in $\mathbb{C}^{2}$.

There is a unique realization of the $Q^{\prime}(\zeta)$ algebra, up to the sign of $\operatorname{det} R$, such that the Hamiltonian operator in both quantizations have the same form, and that realization is

$$
Q^{\prime}\left(\zeta_{k}\right)=\operatorname{det} R \frac{\sigma_{k}}{\sqrt{2}} .
$$

In other words, up to a sign, if the $Q^{\prime}$ quantization is realized in the usual representation by Pauli matrices, $\hat{H}_{F}$ is given by the operator

$$
\hat{H}_{F}=\frac{1}{2} \boldsymbol{\sigma} \cdot \mathbf{F} .
$$

Thus, starting from this requirement, one fixes the isometry $\mathcal{M}$ that will give (34) from (31), and because of the result (23), the $\eta$-inner product is also fixed. As a result, the $Q^{\prime}$-quantization of $H_{F}$ will give the operator (34). Furthermore, one sees from this procedure that the isometry $\mathcal{M}$ is unique. In Sec. IIIB we will provide a systematic way of constructing the isometry.

In conclusion, $\hat{H}_{B}$ describes a quantum theory of a spin system interacting with a real field $\mathbf{B}$, such that $\hat{H}_{B}=\hat{H}_{B}^{\dagger}$. At the same time, $\hat{H}_{F}$ describes a quantum theory of a spin system interacting with a complex field $\mathbf{F}($ with $\operatorname{Im}(\mathbf{F}) \neq 0$ ), such that $\hat{H}_{F}=\hat{H}_{F}^{+}$. In this sense, what we have achieved so far is to connect the description of a nonrelativistic spinning particle under a real field $\mathbf{B}$ with another one with a complex field $\mathbf{F}$, such that the respective Hamiltonians are real under their classic involutions, while the corresponding operators are symmetric (or Hermitian) with respect to the inner products of the Hilbert spaces whereupon they act. Both fields are connected by the complex canonical transformation $R$ by Eq. (18) which implies the important algebraic relation (19).

An important remark following from Eq. (19) should be stressed here. The condition $F^{2} \in \mathbb{R}_{+}$is exactly the condition (7) that the quantum Hamiltonian needs to fulfill so that it is pseudo-Hermitian. In other words, the existence of a real field $\mathbf{B}$, canonically related to a field $\mathbf{F}$ with $\operatorname{Im}(\mathbf{F}) \neq 0$, ensures the reality of the spectrum of $\hat{H}_{F}$, according to Eq. (6). This result implies in the existence of a metric operator $\eta$ that renders $\hat{H}_{F}$ Hermitian. Furthermore, the same canonical transformation connects the two pseudoclassical models whose Hamiltonians are real with respect to the corresponding involutions.

\section{B. Canonical limit and classical correspondence}

Unlike the usual description of pseudo-Hermitian theories, where the metric operator is not unique, we have seen in Sec. IIIA that the metric derived from the isometry (24) is actually unique. We present in this section a schematic way to construct this metric operator, which we call "the canonical limit." 
Besides giving the explicit form of the metric, the canonical limit also furnishes a physical interpretation to our pseudo-Hermitian setup. Vectors related by the isometry $\mathcal{M}$ describe the same physical system. In other words, the Hilbert spaces $\mathcal{H} \simeq\left(\mathbb{C}^{2},\langle\cdot, \cdot\rangle\right)$ and $\mathcal{H}_{\eta} \simeq\left(\mathbb{C}^{2},\langle\cdot, \cdot\rangle_{\eta}\right)$ represent two physically equivalent quantum descriptions (quantizations) of the same classical model, with two classical description that differ by a canonical transformation. Therefore, in order to give correct measurable results, the states must be handled with the appropriate metric.

Let us consider an orthonormal basis $\left\{\phi_{ \pm}\right\}$in $\mathcal{H}$. So, the states $\phi_{ \pm} \in \mathcal{H}$ were prepared (or measured) by the observer associated with the canonical metric in his quantum description. While the states

$$
\phi_{ \pm}^{\prime}=\mathcal{M} \phi_{ \pm}
$$

were prepared by an observer associated with the $\eta$ metric. The states $\left\{\phi_{ \pm}^{\prime}\right\}$ form a orthonormal basis of $\mathcal{H}_{\eta}$. One observer does not agree about the orthogonality of the states prepared by the other. Thus these observers are using different measurement apparatus to construct the quantum description (of the same system). The use of the canonical metric on the state $\phi_{ \pm}^{\prime}$ (or the metric $\eta$ on $\phi_{ \pm}$) is physically meaningless. In the present work, the states whose probabilities must be calculated with the $\eta$ metric are denoted by primes. The physical description by the observer associated with the metric $\eta$ is compatible with the presence of an (effective) complex field $\mathbf{F}$ and the observer associated with the canonical metric measures a real field $\mathbf{B}$. In other words, we distinguish the observables $\hat{H}_{F}: \mathcal{H}_{\eta} \rightarrow \mathcal{H}_{\eta}$ and $\hat{H}_{B}: \mathcal{H} \rightarrow \mathcal{H}$. For every operator $A$ acting on $\mathcal{H}$ there is an equivalent operator $A^{\prime}=\mathcal{M A M}^{-1}$ acting on $\mathcal{H}_{\eta}$.

The classical and quantum descriptions of both observers, especially their notion of orthogonality, must coincide when $\operatorname{Im}(\mathbf{F}) \rightarrow 0$. To achieve this requirement it is necessary to choose $\phi_{ \pm}^{\prime}$ and $\phi_{ \pm}$in Eq. (35) to be, respectively, the eigenvectors of $\hat{H}_{F}=\hat{H}_{F}^{+}$and $\hat{H}_{B}=\hat{H}_{B}^{\dagger}$.

Let us consider with more attention the $\operatorname{limit} \operatorname{Im}(\mathbf{F}) \rightarrow 0$. Following the proposal presented in Ref. [39], we postulate that there are three real dimensionless parameters $\left\{\alpha_{i}\right\}$ such that

$$
\operatorname{Im}\left(F_{i}\right)=\alpha_{i} V_{i} .
$$

The parameters $\left\{\alpha_{i}\right\}$ measure how far the Hamiltonian $\hat{H}_{F}$ is from being canonically Hermitian. Thus, we are interested in systems where the canonical Hermiticity of $\hat{H}_{F}$ is broken continuously, namely, with a well-defined limit $\alpha_{i} \rightarrow 0$. This is a reasonable requirement considering future applications in concrete physical scenarios, where the $\left\{\alpha_{i}\right\}$ are expressed in terms of measurable quantities.

Considering (36), $\hat{H}_{F}$ becomes Hermitian with respect to the canonical inner product, and both theories (defined by $\hat{H}_{B}$ and $\hat{H}_{F}$ ) will differ at most by a unitary transformation. To implement this requirement, for a given $\mathbf{F}$, we choose the real field $\mathbf{B}$ such that

$$
\lim _{\alpha_{i} \rightarrow 0} \mathbf{F}=\lim _{\alpha_{i} \rightarrow 0} \mathbf{B} \in \mathbb{R}^{3} .
$$

We stress that, due to relation (18), the real-valued field $\mathbf{B}$ also depends on $\left\{\alpha_{i}\right\}$. Thus, in order to relate $\mathbf{F}$ and $\mathbf{B}$ in the regime of vanishing $\alpha_{i}$, the limit $\alpha_{i} \rightarrow 0$ must be considered for both fields in Eq. (37). As we will see in a future example, Eq. (37) gives us a prescription such that, when $\alpha_{i} \rightarrow 0$,

$$
\phi_{ \pm}^{\prime} \rightarrow \phi_{ \pm} \Longrightarrow \mathcal{M} \rightarrow \mathbb{I} \Longrightarrow \eta \rightarrow \mathbb{I} .
$$

In summary, the canonical limit is defined to be the prescription (37), together with the unique isometry which defines $\eta$ and relates the eigenvectors of $\hat{H}_{F}$ and $\hat{H}_{B}$.

We turn now our attention to the classical correspondence of two quantum theories: one with a non-Hermitian Hamiltonian, and another with a Hermitian Hamiltonian. From now on we will assume that non-Hermitian operators are those for which there is no inner product with respect to which they are Hermitian.

We construct the classical correspondence by taking mean values of operators. The dynamical variables are real numbers that we expect to be related with the measurable behavior of the system. As will see, for non-Hermitian Hamiltonians this averaging procedure does not recover the classical equations of motion. On the other hand, the classical equations of motion are recovered for pseudo-Hermitian Hamiltonians.

To show the above statement, let us first consider the following non-Hermitian Hamiltonian $\hat{H}$,

$$
\hat{H}=\frac{1}{2} \boldsymbol{\sigma} \cdot[\operatorname{Re}(\mathbf{F})+\mathrm{i} \operatorname{Im}(\mathbf{F})],
$$

which is non-Hermitian by construction since its eigenvalues are not real. We generally define the classical correspondence as the normalized mean value (with the appropriate inner product) of the spin operators $\left\{\sigma_{i}\right\}$; that is,

$$
n_{i} \equiv \frac{\left\langle\psi, \sigma_{i} \psi\right\rangle}{\langle\psi, \psi\rangle}, \mathbf{n}^{2}=1 .
$$


In the present case, because the Hamiltonian is non-Hermitian and there is no suitable inner product, we used the canonical inner product. For $\psi$ a solution of the time-dependent Schrödinger equation, we have

$$
\dot{n}_{i}=\frac{1}{\langle\psi, \psi\rangle}\left[\mathrm{i}\left\langle\psi,\left(\hat{H}^{\dagger} \sigma_{i}-\sigma_{i} \hat{H}\right) \psi\right\rangle-n_{i} \frac{d}{d t}\langle\psi, \psi\rangle\right],
$$

or, in a vector notation,

$$
\dot{\mathbf{n}}=-\mathbf{n} \times \operatorname{Re}(\mathbf{F})-\mathbf{n} \times[\mathbf{n} \times \operatorname{Im}(\mathbf{F})] .
$$

It follows that, when the external field is real, that is, when $\operatorname{Im}(\mathbf{F})=0$, relation (42) coincides with Feynman's results in Ref. [40]. Also, in this case the result (42) reproduces the precession equation (10) of the pseudoclassical theory. However, for $\operatorname{Im}(\mathbf{F}) \neq 0$, Eq. (42) has an additional term that leads to damping of the dynamics of $\mathbf{n}$.

The damping term cannot be obtained classically from the Lagrangian (8) simply taking the external field to have imaginary entries from the very start. The reason is that real and complex fields provide the same equation of motion (10).

At this point, we should mention that, when dealing with a real field $\mathbf{B}$, one has the usual physical interpretation for the SE (1), that is, of a charged particle interacting with an external magnetic field. However, when dealing with a complex field, this notion does not hold. Therefore, in order to give a physical meaning for a complex field, we can look at Eq. (42) as

$$
\dot{\mathbf{n}}=-\mathbf{n} \times \mathbf{F}_{\text {eff }} \text {, with } \mathbf{F}_{\text {eff }}=\operatorname{Re}(\mathbf{F})+\mathbf{n} \times \operatorname{Im}(\mathbf{F})
$$

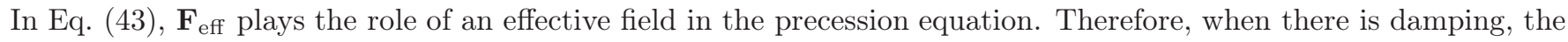
system interacts with the environment in such manner that all the resulting combinations of external and internal fields produce an effective field, which can be represented as a complex external field. In the following section we will give a concrete example.

Consider now the case where $\hat{H}$ is pseudo-Hermitian and therefore $F^{2} \in \mathbb{R}_{+}$. We will show that in this case the theory is unitary, and there are no damping terms in the equations of motion. Let $\langle\cdot, \cdot\rangle_{\eta}$ be the inner product with respect to which $\hat{H}$ is Hermitian. Then the classical correspondence gives

$$
n_{i}(t)=\frac{\left\langle\psi, \sigma_{i} \psi\right\rangle_{\eta}}{\langle\psi, \psi\rangle_{\eta}}=\left\langle\psi, \sigma_{i} \psi\right\rangle_{\eta}
$$

rather than Eq. (40). In this case $\hat{H}^{+}=\hat{H}$ and we have

$$
\dot{n}_{i}(t)=\mathrm{i}\left\langle\psi,\left[\hat{H}, \sigma_{i}\right] \psi\right\rangle_{\eta}=-\varepsilon_{i j k} n_{j}(t) F_{k},
$$

or, in a vector notation,

$$
\dot{\mathbf{n}}=-\mathbf{n} \times \mathbf{F}
$$

The previous equation corresponds to the pseudoclassical equations of motion (10) even when the external field has an imaginary part. The pseudoclassical equations of motion are recovered from the classical correspondence with the identification $\mathbf{n} \rightarrow \boldsymbol{\zeta}$.

We conclude that a non-Hermitian Hamiltonian does indeed describe damping. On the other hand, when the Hamiltonian is pseudo-Hermitian, the external field fulfills the condition (19) and the system does not present a damping behavior. In particular, starting with a non-Hermitian Hamiltonian, we can change the parameters of the effective field (43) such that the condition $F^{2} \in \mathbb{R}_{+}$(with $\operatorname{Im}(F) \neq 0$ ) is satisfied. In this case, there is a configuration of $\mathbf{F}$ such that the damping is completely suppressed. In the following we use this property to propose a possible measurable effect. For $F^{2} \in \mathbb{R}_{+}$, we can summarize the results in the commutative diagram presented in Fig. 1 . We emphasize that the classical-correspondence map in the diagram means that we are able to formally obtain the pseudoclassical equations of motion after the identification of $\mathbf{n}$ with corresponding Grassmann variable, either $\boldsymbol{\xi}$ or $\zeta$.

To consolidate the physical meaning to this correspondence, as well as the physical interpretation of a complex field, let us introduce a concrete scenario in the next section. 


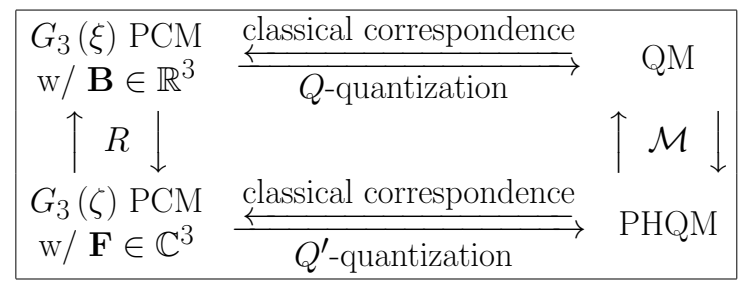

Figure 1. Commutative diagram illustrating the classical-quantum correspondence proposed. PCM denotes pseudoclassical mechanics, QM is short for the quantum theory with Hamiltonian $Q\left(H_{B}\right)$ and Hilbert space $\mathcal{H} \simeq\left(\mathbb{C}^{2},\langle\cdot, \cdot\rangle\right)$, while PHQM is short for the quantum theory with Hamiltonian $Q^{\prime}\left(H_{F}\right)$ and Hilbert space $\mathcal{H}_{\eta} \simeq\left(\mathbb{C}^{2},\langle\cdot, \cdot\rangle_{\eta}\right)$.

\section{PHYSICAL REALIZATION IN THE RABI PROBLEM}

\section{A. Preliminary results}

Now, we present some explicit examples and physical realizations for the previous development, by considering the simplified case when

$$
F_{2}=B_{2}=0 .
$$

As we will see, this particular restriction captures the essential points to be studied in the present work.

Let us examine the following matrix

$$
R=\frac{1}{B_{1}^{2}+B_{3}^{2}}\left(\begin{array}{ccc}
F_{1} B_{1}-B_{3} F_{3} & 0 & F_{1} B_{3}+B_{1} F_{3} \\
0 & -B_{1}^{2}-B_{3}^{2} & 0 \\
F_{1} B_{3}+B_{1} F_{3} & 0 & -\left(F_{1} B_{1}-B_{3} F_{3}\right)
\end{array}\right) .
$$

As one can explicitly check, $R \in S O(3, \mathbb{C})$ for arbitrary complex vectors $\mathbf{F}$ and $\mathbf{B}$ is an explicit solution to the equation $F_{k}=R_{k l} B_{l}$. In other words, $\operatorname{det}(R)=1$ and $R$ preserves the symplectic structure (A12) commented in Appendix In the particular case (48), one has additionally $R=R^{-1}$. Moreover, one can show that equation (19) under the restriction (47),

$$
F_{1}^{2}+F_{3}^{2}=B_{1}^{2}+B_{3}^{2},
$$

is a sufficient condition for the existence of $R$. As shown in Sec. II for $\mathbf{B} \in \mathbb{R}^{3}$, the Hamiltonians

$$
H_{B}(\xi)=-\mathrm{i}\left(B_{1} \xi_{2} \xi_{3}+B_{3} \xi_{1} \xi_{3}\right) \text { and } H_{F}(\zeta)=-\mathrm{i}\left(F_{1} \zeta_{2} \zeta_{3}+F_{3} \zeta_{1} \zeta_{2}\right)
$$

are real in the sense of the involutions,

$$
H_{B}(\xi)=H_{B}^{*}(\xi) \text { and } H_{F}(\zeta)=H_{F}^{+}(\zeta) .
$$

Following our prescription for the canonical limit, we now use the eigenvectors of $\hat{H}_{B}$ and $\hat{H}_{F}$ in order to construct the metric operator $\eta$. Maintaining the convention of using primes to indicate the states whose probabilities must be calculated with the $\eta$ metric, we write the eigenvectors $\phi_{ \pm}^{\prime}$ of $\hat{H}_{F}$, with eigenvalues $E_{F \pm}$, as

$$
\phi_{ \pm}^{\prime}=\frac{1}{F_{1}}\left(\begin{array}{c}
F_{3} \pm E_{F} \\
F_{1}
\end{array}\right), E_{F \pm}= \pm \frac{E_{F}}{2}= \pm \frac{1}{2} \sqrt{F_{1}^{2}+F_{3}^{2}},
$$

and the eigenvector $\phi_{ \pm}$of $\hat{H}_{B}$, with eigenvalues $E_{B \pm}$, as

$$
\phi_{ \pm}=\frac{1}{B_{1}}\left(\begin{array}{c}
B_{3} \pm E_{B} \\
B_{1}
\end{array}\right), E_{B \pm}= \pm \frac{E_{B}}{2}= \pm \frac{1}{2} \sqrt{B_{1}^{2}+B_{3}^{2}} .
$$

From Eq. (49) we see that $E_{F}=E_{B} \equiv E$. The isometry can be read off from relation (35) for the eigenvector $\phi_{ \pm}$and $\phi_{ \pm}^{\prime}$,

$$
\mathcal{M}=\frac{1}{F_{1}}\left(\begin{array}{cc}
B_{1} & F_{3}-B_{3} \\
0 & F_{1}
\end{array}\right)
$$


and the metric operator (23) in $\mathcal{H}_{\eta}$ will be given by

$$
\eta=\frac{1}{B_{1}^{2}}\left(\begin{array}{cc}
\left|F_{1}\right|^{2} & \bar{F}_{1}\left(B_{3}-F_{3}\right) \\
F_{1}\left(B_{3}-\bar{F}_{3}\right) & B_{1}^{2}+\left|B_{3}-F_{3}\right|^{2}
\end{array}\right) .
$$

As expected from the general theory, one has the Hermiticity conditions $\hat{H}_{B}=\hat{H}_{B}^{\dagger}$ and $\hat{H}_{F}=\hat{H}_{F}^{+}$. Besides, by the canonical limit, if $\mathbf{B}=\mathbf{F}$ we have $\mathcal{M}=\mathbb{I}, \eta=\mathbb{I}, \hat{H}_{B}=\hat{H}_{F}$.

Assuming that the operator $\hat{H}_{F}$ is time-independent, the dynamics is simply obtained by exponentiation of $\hat{H}_{F}$. For instance, if one wishes to evaluate a transition amplitude between the eigenvectors of $\sigma_{3} \psi_{ \pm}= \pm \psi_{ \pm}$, that is, the states of "spin-up" $\psi_{+}$and "spin-down" $\psi_{-}$in $\mathcal{H}$, we can construct the corresponding states in $\mathcal{H}_{\eta}$ using the isometry $\mathcal{M}$. This transition amplitude can be written as

$$
\left\langle\psi_{+}^{\prime}, \psi^{\prime}(t)\right\rangle_{\eta}=\left\langle\psi_{+}^{\prime}, \exp \left(-\mathrm{i} \hat{H}_{F} t\right) \psi_{-}^{\prime}\right\rangle_{\eta}=-\mathrm{i} \frac{B_{1}}{E} \sin \left(\frac{E}{2} t\right),
$$

where $\psi_{ \pm}^{\prime}=\mathcal{M} \psi_{ \pm}$. We note the oscillatory behavior of (56), which is a characteristic property of unitary theories.

Let us illustrate the above with the example of the Rabi oscillations in an assumed damped two-level system [41]

$$
F_{1}=V \in \mathbb{R}, F_{2}=0, F_{3}=\mathrm{i} \alpha, \alpha^{2}<V^{2} .
$$

The $\mathbf{F}$ field can be obtained from the canonical transformation (48) starting from any one of the following real $\mathbf{B}$ fields and rotations thereof

$$
\begin{aligned}
& B_{1}=B_{2}=0, B_{3}= \pm \sqrt{V^{2}-\alpha^{2}}, \\
& B_{1}= \pm \sqrt{V^{2}-\alpha^{2}}, B_{2}=B_{3}=0 .
\end{aligned}
$$

However, the canonical limit (37) implies the specific choice

$$
B_{1}=\operatorname{sgn}(V) \sqrt{V^{2}-\alpha^{2}}, B_{2}=B_{3}=0 .
$$

As one can directly check, the Hamiltonian $\hat{H}_{F}$ is Hermitian according to the metric (55), that is, it satisfies $\hat{H}_{F}=$ $\eta^{-1} \hat{H}_{F}^{\dagger} \eta$. Given the configuration for $\mathbf{B}$ in (59), one can verify the canonical limit (37) $\lim _{\alpha \rightarrow 0} \eta=\mathbb{I}$. The transition amplitude (56) between spin-up and spin-down states reads

$$
\left\langle\psi_{+}^{\prime}, \exp \left(-\mathrm{i} \hat{H}_{F} t\right) \psi_{-}^{\prime}\right\rangle_{\eta}=-\mathrm{i}[\operatorname{sgn}(V)] \sin \left(\frac{\sqrt{V^{2}-\alpha^{2}}}{2} t\right) .
$$

Apart from the factor 1/2, due to our particular choice of constants (see footnote 1), the oscillation frequency of the amplitude (60) agrees with the one in Ref. [41]. However, unlike in Ref. [41], here the evolution is unitary and states do not lose their normalization condition under time evolution. In general, there is a critical value $\alpha_{c}$ of $\alpha$ for which $\operatorname{Im}(E)=\left(V^{2}-\alpha^{2}\right)^{1 / 2} \neq 0$ if $\alpha>\alpha_{c}$. In the illustrative example presented in this section, this critical value $\alpha_{c}=V$ can be read from Eq. (57). In some descriptions, the value $\alpha_{c}$ can be associated with a possible phase transition [39]. In this article, conditions (49) and $E_{F}=E_{B} \in \mathbb{R}$ are assumed.

\section{B. Rabi problem and the Gilbert damping term}

Let us now consider the more elaborate Rabi problem [1, 2]. This is a two-level system, consisting of a single electron fixed in the space, in interaction with an external magnetic field given by

$$
\mathbf{B}_{R}=\left(B \cos (\omega t), B \sin (\omega t), B_{z}\right),
$$

with $B, B_{z}$ and $\omega$ real constants. We can eliminate the second component of the $\mathbf{B}$ field by changing to a rotating reference frame with the help of the rotation

$$
R_{z}(\omega t)=\exp \left(\frac{\mathrm{i} \omega \sigma_{3} t}{2}\right)
$$


In this rotating reference frame we have

$$
B_{1}=B, B_{2}=0, B_{3}=\delta, \delta=B_{z}-\omega,
$$

and time-independent Hamiltonian

$$
\hat{H}_{R}=\frac{1}{2}\left(\delta \sigma_{3}+B \sigma_{1}\right)
$$

The transition amplitude between spin-up and spin-down states $\left(\sigma_{3} \psi_{ \pm}= \pm \psi_{ \pm}\right)$is given by the Rabi oscillations

$$
\left\langle\psi_{+}, \exp \left(-\mathrm{i} \hat{H}_{R} t\right) \psi_{-}\right\rangle=-\mathrm{i} \frac{B}{\Omega_{R}} \sin \left(\frac{\Omega_{R}}{2} t\right), \Omega_{R}^{2}=B^{2}+\delta^{2}
$$

The $\delta$ factor is called detuning, while $\Omega_{R}$ and $\omega=B_{z}$ denote the Rabi frequency and resonance frequency respectively.

As we have seen in Sec. IIIB, a damped precession is characteristic of a nonunitary evolution. Indeed, we can see that the damping term in Eq. (42) arises exactly from the imaginary part of field, which is what breaks the hermiticity of the Hamiltonian. Therefore, one can consider a damped version of the Rabi problem by introducing an imaginary term in the field (63). For this reason, we choose the external field to be

$$
F_{1}=\frac{1+\mathrm{i} \alpha}{1+\alpha^{2}} B, F_{2}=0, F_{3}=\frac{1+\mathrm{i} \alpha}{1+\alpha^{2}} B_{z}-\omega, \alpha \in \mathbb{R} .
$$

In the limit $\alpha \rightarrow 0$, this field configuration reduces to the original Rabi problem characterized by (63) in the rotating frame. For arbitrary values of the parameters $B, B_{z}, \omega, \alpha$, the Hamiltonian $\hat{H}_{F}$ is non-Hermitian, resulting in a damped behavior.

The time-dependent field configuration for the damped Rabi setup in the nonrotating frame is

$$
\mathbf{F}_{R}=\left(F_{1} \cos (\omega t), F_{1} \sin (\omega t), F_{3}\right)=\frac{1+\mathrm{i} \alpha}{1+\alpha^{2}} \mathbf{B}_{R}
$$

which reduces to the original Rabi problem described by $\mathbf{B}_{R}$ in (61) when $\alpha$ is set to zero. We can now obtain the classical correspondence, which we interpret as the behavior of the damped system as actually measured. Substituting the field configuration (67) in Eq. (42), we have

$$
\dot{\mathbf{n}}=-\frac{1}{1+\alpha^{2}} \mathbf{n} \times \mathbf{B}_{R}-\frac{\alpha}{1+\alpha^{2}} \mathbf{n} \times\left(\mathbf{n} \times \mathbf{B}_{R}\right) .
$$

A physical interpretation can now be provided for the parameter $\alpha$. The above equation describes a damped precession of the magnetic moment. As is well known, this phenomenon can be adequately described by the LandauLifshitz-Gilbert (LLG) equation [42], which consists of introducing an ad hoc term in the undamped equation of motion. The LLG equation, for the unit magnetization $\hat{\mathbf{n}}$, subject to a magnetic field $\mathbf{B}$, has the form [43]

$$
\frac{d \hat{\mathbf{n}}}{d t}=-\frac{1}{1+\alpha^{2}} \hat{\mathbf{n}} \times \mathbf{B}-\frac{\alpha}{1+\alpha^{2}} \hat{\mathbf{n}} \times(\hat{\mathbf{n}} \times \mathbf{B}),
$$

where $\alpha$ is the Gilbert damping parameter. By comparing the LLG equation (69) with the relation (68) obtained via classical correspondence, we see that the $\alpha$ parameter introduced in Eq. (66) can be identified with the Gilbert damping parameter.

Even though we have just addressed the damped Rabi problem, the identification of $\alpha$ with the Gilbert damping term is valid for a general effective field $\mathbf{F}$ in the form

$$
\mathbf{F}=\frac{1+\mathrm{i} \alpha}{1+\alpha^{2}} \mathbf{B}
$$

for any $\mathbf{B} \in \mathbb{R}^{3}$. This follows from the fact that the classic correspondence equation (42) is exactly the LLG equation for the field configuration (70). 


\section{The pseudo-Hermitian version of the Rabi problem}

In this section we choose the parameters $B, B_{z}, \omega$ and $\alpha$ such that the restriction (49) is satisfied, so that $\hat{H}_{F}$ is (pseudo) Hermitian, $\hat{H}_{F}^{+}=\hat{H}_{F}$. We introduce the notation

$$
F \equiv F_{1}=\frac{1+\mathrm{i} \alpha}{1+\alpha^{2}} B \text { and } \Delta \equiv F_{3}=\frac{1+\mathrm{i} \alpha}{1+\alpha^{2}} B_{z}-\omega,
$$

to label the field components satisfying the condition (49). Now the classical Hamiltonian

$$
H_{F}=-\mathrm{i}\left(F \zeta_{2} \zeta_{3}+\Delta \zeta_{1} \zeta_{2}\right)
$$

is real $\left(H_{F}^{+}=H_{F}\right)$. The specific choice of parameters can be found from Eq. (17), i.e., from $\operatorname{Im}\left(F^{2}+\Delta^{2}\right)=0$,

$$
B^{2}+\delta^{2}-\alpha^{2} \omega^{2}+\delta \omega\left(1-\alpha^{2}\right)=0
$$

It follows that

$$
F^{2}+\Delta^{2}=-\delta \omega=B_{1}^{2}+B_{3}^{2}
$$

Even though B has not yet been determined, the eigenvalues (53) of $\hat{H}_{B}$ are known, because of relation (49). The Hamiltonian $\hat{H}_{F}$ has the eigenvectors $\phi_{ \pm}^{\prime}$ and eigenvalues $E_{ \pm}$:

$$
\phi_{ \pm}^{\prime}=\frac{1}{F}\left(\begin{array}{c}
\Delta \pm \Omega \\
F
\end{array}\right), E_{ \pm}= \pm \frac{\Omega}{2}, \Omega^{2}=F^{2}+\Delta^{2} .
$$

From Eq. (74), for $\delta \omega>0$ the eigenvalues are purely imaginary, however we only consider the case where $\delta \omega<0$, that is, the case of real eigenvalues. Considering that the limit $\alpha \rightarrow 0$ implies

$$
\Delta \rightarrow \delta, F \rightarrow B, \Omega \rightarrow \Omega_{R}
$$

we use the canonical limit to construct the eigenvectors $\phi_{ \pm}$of $\hat{H}_{B}$, which has the same eigenvalues $E_{ \pm}$:

$$
\phi_{ \pm}=\frac{1}{B}\left(\begin{array}{c}
\delta \pm \Omega_{R} \\
B
\end{array}\right)
$$

After calculating the eigenvectors in (75) and (777), one can determine the isometry $\mathcal{M}$,

$$
\mathcal{M}=\frac{1}{F \Omega_{R}}\left(\begin{array}{cc}
B \Omega & \Delta \Omega_{R}-\delta \Omega \\
0 & F \Omega_{R}
\end{array}\right),
$$

and the metric operator $\eta$,

$$
\eta=\frac{1}{B^{2} \Omega^{2}}\left(\begin{array}{cc}
|F|^{2} \Omega_{R}^{2} & \bar{F} \Omega_{R}\left(\delta \Omega-\Delta \Omega_{R}\right) \\
F \Omega_{R}\left(\delta \Omega-\bar{\Delta} \Omega_{R}\right) & B^{2} \Omega^{2}+\left|\delta \Omega-\Delta \Omega_{R}\right|^{2}
\end{array}\right)
$$

The expression for $\eta$ in (79) satisfies the canonical limit $\eta \rightarrow \mathbb{I}$ when $\alpha \rightarrow 0$. The explicit form of $\hat{H}_{B}$ can be obtained from result (26), i.e., $\hat{H}_{B}=\mathcal{M}^{-1} \hat{H}_{F} \mathcal{M}$. Moreover, one can determine the $\mathbf{B}$ field,

$$
\mathbf{B}=\frac{\Omega}{\Omega_{R}}(B, 0, \delta),
$$

and the canonical transformation $R$ from Eq. (48).

To obtain the pseudo-Hermitian version of the damped Rabi problem in the original (nonrotating) frame, one must rotate back the reference frame with the rotation $R_{z}^{\prime}=\mathcal{M} R_{z} \mathcal{M}^{-1}$, where $R_{z}(-\omega)$ is given in Eq. (62), that is,

$$
\hat{H}_{F}^{\prime}=\mathrm{i} \frac{\partial R_{z}^{\prime}}{\partial t}\left(R_{z}^{\prime}\right)^{-1}+R_{z}^{\prime} \hat{H}_{F}\left(R_{z}^{\prime}\right)^{-1}=\mathcal{M} \hat{H}_{B}^{\prime} \mathcal{M}^{-1}
$$

with

$$
\hat{H}_{B}^{\prime}=\frac{1}{2 \Omega_{R}}\left(\begin{array}{cc}
\delta \Omega+\omega \Omega_{R} & B \Omega \exp (-i \omega t) \\
B \Omega \exp (i \omega t) & -\left(\delta \Omega+\omega \Omega_{R}\right)
\end{array}\right) .
$$


As expected, $\hat{H}_{B}^{\prime}$ is the field obtained from (80) by the usual rotation (62). The Hamiltonian $\hat{H}_{F}^{\prime}$ keeps its pseudoHermiticity. In the canonical limit, not only we verify that $\hat{H}_{F}^{\prime} \rightarrow \hat{H}_{B}^{\prime}$, but we also recover the Hamiltonian associated with the Rabi problem in the nonrotating frame (61).

Let us consider the dynamics of this model. Using Eq. (79) we can determine the transition amplitude (56) between spin-up and spin-down states,

$$
\left\langle\psi_{+}^{\prime}, \exp \left(-\mathrm{i} \hat{H}_{F} t\right) \psi_{-}^{\prime}\right\rangle_{\eta}=-\mathrm{i} \frac{B}{\Omega_{R}} \sin \left(\frac{\Omega}{2} t\right) .
$$

The frequency $\omega=B_{z}(\delta=0 \Rightarrow \Omega=0)$ represents a critical point, which can be associated with symmetry breaking. From relations (74) and (73) we have

$$
\Omega^{2}=\left\{\begin{array}{l}
\Omega_{R}^{2}+\frac{\alpha^{2}}{1-\alpha^{2}}\left(\Omega_{R}^{2}-\omega^{2}\right) \text { for } \alpha \neq \pm 1 \\
\left|\delta \Omega_{R}\right| \text { for } \alpha= \pm 1
\end{array} .\right.
$$

In summary, when condition (49) holds, the theory is unitary, and there is no damping term in the equations of motion.

Previous results can furnish possible measurable effects. The main point is that $\hat{H}_{F}$ is non-Hermitian, and thus there would be a damping term in the equations of motion for any value of the external field, except if (73) is valid. When condition (73) is satisfied, the damping effect disappears and the evolution of the system becomes unitary. From Eq. (74) we see that, when $\omega>0$, the pseudo-Hermitian regime can only be reached for $\delta<0$. This means that it is not possible to suppress damping with a frequency below the resonance frequency of the usual Rabi problem. In addition, we can use Eq. (73) to determine, for example, $B$ as a function of the other parameters:

$$
B^{2}=B_{z}\left[\omega\left(1+\alpha^{2}\right)-B_{z}\right] \text { for } B, B_{z}, \alpha \neq 0 .
$$

We interpret the condition (85) as the configuration of the $\mathbf{B}$ field which injects energy in the system at the same rate the system dissipates energy. In this case, the damping effect is completely suppressed and the classical limit is again a precession movement described by (46), and not by the LLG equation (69).

\section{Experimental viability}

We now consider the experimental implementation of the ideas introduced. In the arrangement presented in the previous section, the suppression of damping may be identified with the so-called steady-state precession or selfsustained precessional motion (SSPM) [44], where an effective field cancels the spin damping, generating a constant angle precession. Although the direct measurement of the local magnetization is a challenge in an actual laboratory experiment, the spin dynamics can be controlled and determined by a current-induced magnetization. The magnetization itself is controlled by a combination of the external field and the current electrons spin-transfer-induced precession of magnetization.

By varying the applied magnetic field and the direct current irradiated with high-frequency microwaves, it is possible to control the precession frequency, obtaining values from the order of $1 \mathrm{GHz}$ up to $100 \mathrm{GHz}$ or larger. In fact, the irradiation induces microwave currents, which are equivalent to the arrangement where currents are fed to the contact through electric leads [45].

The high-frequency dynamics of the magnetization can be measured directly by detecting high-frequency voltage oscillations [46]. This current-induced magnetization is different from the setup we have considered, since it is related to spin-transfer torque. However, we are interested in the macrospin model with uniform magnetization. In this case, a description of the magnetization dynamics using a modified LLG equation that can be written in the form (43) is largely equivalent [47].

A concrete example where the SSPM can be detected are the so-called spin valves. A simple spin valve is formed by two conducting magnetic materials, the free layer and the reference layer, separated by a (metallic or insulator) spacer. The electric resistance of the device can be controlled through the relative alignment of the magnetization in the layers. The spins of the electron current are adjusted by a hard and thick magnetic layer called the reference layer, while the layer where magnetization will be manipulated is called the free layer. A typical setup uses a pinned antiferromagnet reference layer coupled to a ferromagnetic free layer.

In this device an applied current generates a spin torque that, under specific conditions, can cancel the Gilbert damping term in the LLG equation resulting inan undamped precession (46). Namely, for the spin valve, the modified LLG equation assumes the form [44]

$$
\frac{d \hat{\mathbf{n}}}{d t}=-\frac{1}{1+\alpha^{2}} \hat{\mathbf{n}} \times \mathbf{B}-\frac{\alpha}{1+\alpha^{2}} \hat{\mathbf{n}} \times(\hat{\mathbf{n}} \times \mathbf{B})+a \hat{\mathbf{n}} \times(\hat{\mathbf{n}} \times \mathbf{P}),
$$


where $\mathbf{P}$ is a fixed unit vector in the magnetization direction of the pinned layer in the spin valve and the torque coefficient $a$ depends on the applied current and may also depend on the angle between the magnetization directions of the pinned and free layers [48]. The effective field B in Eq. (86) is the combination of the exchange field, the anisotropy field, the demagnetization field, and the applied external field. A qualitative and quantitative discussion of Eq. (86) can be found in Refs. [44, 47] and references therein.

It is possible to show [43] that the effect of the new term in the modified LLG equation (86) is simply to change the effective magnetic field to

$$
\mathbf{B}=\left(B_{x}, B_{y}, B_{z}\right) \longrightarrow\left(B_{x}-\mathrm{i} a P_{x}, B_{y}+\mathrm{i} a P_{y}, B_{z}+\mathrm{i} a P_{z}\right)
$$

That is, the effect of the spin-transfer-induced is equivalent to the addition of an imaginary term to the magnetic field already present in the original LLG equation. Therefore, by choosing the magnetization direction of the reference layer in the $z$-direction, that is $\mathbf{P}=(0,0,1)$, the spin-transfer-induced effect can be taken into account with the change

$$
B_{z} \longrightarrow B_{z}+\mathrm{i} a
$$

Repeating our previous development using the new field $B_{z}$, it is straightforward to show that condition (85) becomes

$$
B^{2}=\omega\left(1+\alpha^{2}\right) B_{z}-B_{z}^{2}+\frac{a}{\alpha}\left[\alpha a-B_{z}\left(1-\alpha^{2}\right)+\omega\left(1+\alpha^{2}\right)\right] .
$$

Constraint (89) reduces to (85) for $a=0$.

The SSPM solution of the modified LLG equation (86) can be calculated using a perturbative method based on Melkinov integrals [49]. The theoretical analysis can be experimentally verified by measurements of voltage oscillations related to the dynamics of the magnetization, as previously discussed. We believe that the parameters of the SSPM solution for the field B, obtained in this experimental setup, can be compared with the constraint (89). This should be a nontrivial test of the formalism developed in the present work.

\section{FINAL REMARKS}

In this work, a classical-quantum correspondence for a pseudo-Hermitian system with finite energy levels is proposed and analyzed. A dictionary connects particles subjected to real and complex fields ( $\mathbf{B}$ and $\mathbf{F})$, related by a canonical transformation. The quantization map ensures Hermiticity of operators, whose symbols are real functions in the respective pseudoclassical phase space. The commutativity of the quantization map relates canonical transformations between symbols to unitary transformations between the corresponding operators. In particular, the Hamiltonians associated with $\mathbf{B}$ and $\mathbf{F}$ are real under their classic involutions, and the corresponding operators are symmetric (or Hermitian) with respect to the inner products of the Hilbert spaces whereupon they act. An important point in our development is the notion that there is no fundamental distinction between Hermitian and pseudo-Hermitian (Hamiltonian) operators, or even between ordinary quantum mechanics and pseudo-Hermitian quantum mechanics for that matter, as long as the relations summarized by Fig. 1 are satisfied. That is, as long as the possibility of a metric redefinition which reestablishes Hermiticity in quantum theory can be seen as a consequence of a proper choice of coordinates in the pseudoclassical theory. The only nontrivial physical statement is that some non-Hermitian operators can become pseudo-Hermitian under certain regimes.

Furthermore, we show that there is a unique isometry between the Hilbert spaces $\left(\mathbb{C}^{n},\langle\cdot, \cdot\rangle\right)$ and $\left(\mathbb{C}^{n},\langle\cdot, \cdot\rangle_{\eta}\right)$ that preserves the representation of the Clifford algebra chosen in both settings (real and complex), implying a unique metric. A systematic way of constructing this metric is provided. In addition, we apply the classical correspondence to the two-level quantum system coupled to a complex field. For non-Hermitian Hamiltonians, this correspondence describes damping and does not recover the classical equations of motion. When the Hamiltonian is pseudo-Hermitian, this correspondence does not imply damping and the classical equations of motion are recovered. Indeed, a common claim in the literature is that a complex Hamiltonian for a spin- $1 / 2$ particle always leads to spin precession with damping. We have shown that this is not necessarylly true. Hamiltonians associated with complex fields, which are non-Hermitian with the usual inner product, do not necessarily generate damping.

As a concrete development, we propose a damped version of the Rabi setup, considering a complex field associated with a non-Hermitian Hamiltonian. We identify the parameter that controls the intensity of the imaginary part as the Gilbert damping parameter of the Landau-Lifshitz-Gilbert equation. In this setup, we find a specific configuration of the parameters where the damping is completely suppressed. In this case, the classical correspondence describes again a precession movement for the spinning particle. We interpret this arrangement as the configuration where the applied field completely compensates the damping effect. It may be identified with the so-called steady-state precession [44, where an external field cancels the spin damping, generating a constant angle precession. The steady-state regime 
could be observed with measurements involving ferromagnetic resonance methods $[\underline{50}$. We believe that the presented developments could be verified in laboratory tests. For instance, using an experimental setup based on spin valves, the modified LLG constraint (89) can be checked. This would be a nontrivial test of the theoretical formalism introduced.

The classical-quantum correspondence for two-level pseudo-Hermitian systems may have practical applications. Precise manipulation of the spin has several technological consequences and a description of damping process is essential in this manipulation. For example, in the emerging technologies of spintronic devices. In nowadays applications, the dynamics of the magnetization in the digital storage process is described by the LLG equation and any deviation from this description should have practical implications. The possibility of suppressing the damping behavior could lead to a faster and more energy-efficient spin manipulation. Phenomena in the steady-state precession regime also have consequences in processes involving magnetic resonance [51].

Recent developments of the pseudo-Hermitian setup suggest interesting perspectives for the theoretical framework presented here. Effects involving non-Hermiticity enhances the dynamics of the topological-phase transitions, bringing up new effects considering the scenarios involving the usual Hermitian framework [52]. Topological properties of the theory can be explored, by evaluating quantities such as the Berry phase. A second-quantization approach of the semiclassical damped Rabi problem proposed in the present work can be investigated following a treatment in the same lines as the one presented in Ref. [53].

Finally, the developed formalism might be extendable to lattice systems [54]. In this case topological phase transitions in the exceptional points could be investigated, as done for optical lattices [55], as well as Anderson localization and mobility edges in non-Hermitian systems [56]. Another topological phenomenon that can be explored in this context is the non-Hermitian skin effect [57]. In this case, the suppression of the damping, described in this work, may have some relationship with the elimination of the non-Hermitian skin effect described in Ref. [26].

\section{ACKNOWLEDGMENTS}

K. R. acknowledges the support of Coordenação de Aperfeiçoamento de Pessoal de Nível Superior (CAPES), Finance Code 001, Brazil; and the National Council for Scientific and Technological Development (CNPq), Brazil, with grant \#141264/2020-9. R. F. acknowledges the support of São Paulo Research Foundation (FAPESP), Brazil, with grant \#2016/03319-6. C. M. acknowledges the support of National Council for Scientific and Technological Development (CNPq), Brazil, with grant \#420878/2016-5.

\section{Appendix A: Pseudoclassical model}

In this appendix we give a brief presentation of a simple nonrelativistic model for a spinning particle in the context of pseudoclassical mechanics. Following [36], one considers a phase-space formulation where dynamical variables are functions on a Grassmann algebra, such that, upon quantization, their Poisson brackets provide the correct commutation relations.

The Grassmann algebra $G_{n}(\xi)$ is an algebra over the complex field $\mathbb{C}$ whose generators $\xi_{i}, i=1, \ldots, n$ satisfy the relations

$$
\xi_{i} \xi_{j}+\xi_{j} \xi_{i}=0
$$

Functions $f(\xi)$ on $G_{n}(\xi)$ are polynomials in the generators $\xi_{i}$. Hence, one can define a derivative operator acting on monomials and, by extension, on functions, as the right-derivatives

$$
\frac{\partial}{\partial \xi_{i}} \xi_{i_{1}} \xi_{i_{2}} \cdots \xi_{i_{k}}=\sum_{j=1}^{k}(-1)^{k-j} \delta_{i i_{j}} \xi_{i_{1}} \xi_{i_{2}} \cdots \xi_{i-1} \xi_{i+1} \cdots \xi_{i_{k}} .
$$

For our purposes, it is enough to consider Grassmann algebras with three generators, that is, $n=3$. Thus, a general function $f(\xi)$ on the Grassmann algebra $G_{3}(\xi)$ is given by

$$
f(\xi)=f_{0}+f_{i} \xi_{i}+f_{i j} \xi_{i} \xi_{j}+\frac{\mathrm{i}}{3 !} k_{f} \varepsilon_{i j k} \xi_{i} \xi_{j} \xi_{k},
$$

where $f_{0}, f_{i}, f_{i j}, k_{f} \in \mathbb{C}$ and $f_{i j}=-f_{j i}$. Odd-parity functions $f$ are sums of homogeneous terms with odd numbers of the Grassmann generators, and we write $P_{f}=1$. Even-parity functions $f$ are those containing even number of generators, and we write $P_{f}=0$. 
A relevant nonrelativistic pseudoclassical model is given by the action

$$
S=\int_{t_{i}}^{t_{f}} L(\xi, \dot{\xi}) d t, L=\frac{\mathrm{i}}{2} \xi_{i} \dot{\xi}_{i}-H(\xi)
$$

where $H(\xi)$ is some even function of the $\left\{\xi_{i}\right\}, P_{H}=0$. One can proceed as in usual mechanics, and define the conjugate momenta

$$
\pi_{i}=\frac{\partial L}{\partial \dot{\xi}_{i}}=\frac{\mathrm{i}}{2} \xi_{i}
$$

with the derivatives always taken from the right, as defined in Eq. A2 . As a result, one finds the canonical Hamiltonian function

$$
H_{c}(\xi, \pi)=H(\xi)+\left(\pi_{i}-\frac{\mathrm{i}}{2} \xi_{i}\right) \dot{\xi}_{i}
$$

There is a natural Poisson bracket in the coordinates $(\xi, \pi)$. Let $f$ and $g$ be functions of the Grassmann variables of definite parity. Then, the Poisson bracket between them is defined as

$$
\{f, g\}=\frac{\partial f}{\partial \xi_{i}} \frac{\partial g}{\partial \pi_{i}}-(-1)^{P_{f} P_{g}} \frac{\partial g}{\partial \xi_{i}} \frac{\partial f}{\partial \pi_{i}}
$$

where derivatives are taken from the right as usual. Thus, the Poisson brackets between the canonical pairs are

$$
\left\{\xi_{i}, \pi_{j}\right\}=\left\{\pi_{j}, \xi_{i}\right\}=\delta_{i j}
$$

It should be noticed that the equations are constraints, which we denote as

$$
\phi_{i}=\pi_{i}-\frac{\mathrm{i}}{2} \xi_{i}
$$

Their conservation in time fixes the velocities $\left\{\dot{\xi}_{i}\right\}$ :

$$
\left\{\phi_{i}, H_{c}\right\}=0 \Longrightarrow \dot{\xi}_{i}=\mathrm{i} \frac{\partial H}{\partial \xi_{i}}
$$

Therefore, according to Dirac's terminology for constrained systems, the model A4 is a second-class theory, that is, there are no first-class constraints and the dynamics is completely determined on the constraint surface $\phi=0$. Following Dirac's quantization scheme for second-class theories [58], we first define the Dirac brackets over the set of second-class constraints $\phi$ as

$$
\{f, g\}_{D(\phi)}=\{f, g\}-\left\{f, \phi_{i}\right\} C_{i j}^{-1}\left\{\phi_{j}, g\right\}
$$

where $C_{i j}^{-1}$ denotes the inverse matrix to $C_{i j}=\left\{\phi_{i}, \phi_{j}\right\}=-\mathrm{i} \delta_{i j}$, and again $f$ and $g$ are parity-definite functions of the Grassmann variables. Thus the nonvanishing Dirac brackets between canonical variables are

$$
\left\{\xi_{i}, \xi_{j}\right\}_{D(\phi)}=-\mathrm{i} \delta_{i j},\left\{\pi_{i}, \pi_{j}\right\}_{D(\phi)}=\frac{\mathrm{i}}{4} \delta_{i j},\left\{\xi_{i}, \pi_{j}\right\}_{D(\phi)}=\frac{1}{2} \delta_{i j} .
$$

One can use the constraints $\phi$ to eliminate the momenta from the description, so that one is only left with coordinates $\xi_{i}$.

\section{Appendix B: Quantization}

Exceptionally in this appendix we restore $\hbar$. Let us define a quantization map $Q: G_{3}(\xi) \rightarrow L(\mathcal{H})$, where $G_{3}(\xi)$ is the Grassmann algebra with generators $\left\{\xi_{i}\right\}_{i=1}^{3}$ and $L(\mathcal{H})$ is the set of bounded linear operators on the Hilbert space $\mathcal{H}=\left(\mathbb{C}^{2},\langle\cdot, \cdot\rangle\right)$. It suffices to define the map on monomials, following the antisymmetrization rule

$$
Q\left(\xi_{1} \xi_{2} \cdots \xi_{n}\right)=\frac{1}{n !} \sum_{\text {perm }}(-1)^{\sigma(\text { perm })} Q\left(\xi_{i_{1}}\right) Q\left(\xi_{i_{2}}\right) \cdots Q\left(\xi_{i_{n}}\right)
$$


and extend it linearly to all functions. Furthermore, the quantization map $Q$ is required to map the unit to the identity in $\mathcal{H}, Q(1)=\mathbb{I}$. In our case, the above requirements imply the following for general classical functions:

$$
Q(f)=f_{0} \mathbb{I}+f_{i} Q\left(\xi_{i}\right)+f_{i j} Q\left(\xi_{i}\right) Q\left(\xi_{j}\right)+\mathrm{i} \frac{1}{3 !} k_{f} \varepsilon_{i j k} Q\left(\xi_{i}\right) Q\left(\xi_{j}\right) Q\left(\xi_{k}\right) .
$$

It should be noticed that the quantization map $Q$ satisfies

$$
f=f^{*} \Longrightarrow\langle x, Q(f) y\rangle=\langle Q(f) x, y\rangle \text {. }
$$

That is, for real functions $f, Q(f)$ is symmetric, $Q^{\dagger}(f)=Q(f)$. The map $Q$ is also required to satisfy the correspondence principle

$$
\{f, h\}_{D(\phi)}=\lim _{\hbar \rightarrow 0} \frac{1}{\mathrm{i} \hbar}[Q(f), Q(h)]
$$

where $[\cdot, \cdot]$ is a $Z_{2}$-graded commutator:

$$
[Q(f), Q(h)]=Q(f) Q(h)-(-1)^{P_{f} P_{h}} Q(h) Q(f)
$$

for all homogeneous functions $f$ and $h$. Thus, one has for the dynamical variables $\left\{\xi_{i}\right\}_{i=1}^{3}$ the basic anticommutation relations

$$
\left[Q\left(\xi_{i}\right), Q\left(\xi_{j}\right)\right]=\hbar \delta_{i j}
$$

The above development is standard procedure on the program of quantization of pseudoclassical models. Details can be found, for example, in reference textbooks [58, 59].

[1] I. I. Rabi, N. F. Ramsey, and J. Schwinger, Use of rotating coordinates in magnetic resonance problems, Rev. Mod. Phys. 26, 167 (1954).

[2] I. I. Rabi, Space quantization in a gyrating magnetic field, Phys. Rev. 51, 652 (1937).

[3] I. Rotter, A non-Hermitian Hamilton operator and the physics of open quantum systems, J. Phys. A 42, 153001 (2009).

[4] P. A. M. Dirac and R. H. Fowler, A theory of electrons and protons, Proc. R. Soc. Lond. A 126, 360 (1930).

[5] W. Pauli, On Dirac's New method of field quantization, Rev. Mod. Phys. 15, 175 (1943).

[6] S. N. Gupta, Theory of longitudinal photons in quantum electrodynamics, Proc. Phys. Soc. A 63, 681 (1950).

[7] K. Bleuler, Eine neue Methode zur Behandlung der longitudinalen und skalaren Photonen, Helv. Phys. Acta 23, 567 (1950).

[8] E. C. G. Sudarshan, Quantum mechanical systems with indefinite metric. I, Phys. Rev. 123, 2183 (1961).

[9] T. D. Lee and G. C. Wick, Negative metric and the unitarity of the S-matrix, Nucl. Phys. B 9, 209 (1969).

[10] F. G. Scholtz, H. B. Geyer, F. J. W. Hahne, Quasi-Hermitian operators in quantum mechanics and the variational principle, Ann. Phys. 213, 74 (1992).

[11] C. M. Bender and S. Boettcher, Real spectra in non-Hermitian Hamiltonians having PT symmetry, Phys. Rev. Lett. 80, 5243 (1998). arXiv: physics/9712001

[12] C. M. Bender, D. C. Brody, and H. F. Jones, Complex extension of quantum mechanics, Phys. Rev. Lett. 89, 270401 (2002). arXiv: quant-ph/0208076.

[13] C. M. Bender, D. C. Brody, H. F. Jones, Must a Hamiltonian be Hermitian?, Am. J. Phys. 71, 1095 (2003). arXiv:hep-th/0303005.

[14] C. M. Bender, J. Brod, A. Refig and M. E. Reuter, The $\mathcal{C}$ operator in $\mathcal{P} \mathcal{T}$-symmetric quantum theories, J. Phys. A 37, 10139 (2004). arXiv:quant-ph/0402026.

[15] C. M. Bender, Making sense of non-Hermitian Hamiltonians, Rep. Prog. Phys. 70, 947 (2007). arXiv:hep-th/0703096.

[16] A. Mostafazadeh, Pseudo-Hermiticity versus $\mathcal{P} \mathcal{T}$-symmetry: the necessary condition for the reality of the spectrum of a non-Hermitian Hamiltonian, J. Math. Phys. 43, 205 (2002). arXiv:math-ph/0107001.

[17] A. Mostafazadeh, Pseudo-Hermiticity versus $\mathcal{P} \mathcal{T}$-symmetry. II. A complete characterization of non-Hermitian Hamiltonians with a real spectrum, J. Math. Phys. 43, 2814 (2002). arXiv:math-ph/0110016.

[18] A. Mostafazadeh, Pseudo-Hermiticity versus $\mathcal{P} \mathcal{T}$-symmetry III: Equivalence of pseudo-Hermiticity and the presence of antilinear symmetries, J. Math. Phys. 43, 3944 (2002). arXiv:math-ph/0203005.

[19] A. Mostafazadeh and Ahmet Batal, Physical aspects of pseudo-Hermitian and $\mathcal{P}$ T-symmetric quantum mechanics, J. Phys. A 37, 11645 (2004). arXiv:quant-ph/0408132

[20] A. Mostafazadeh, Is pseudo-Hermitian quantum mechanics an indefinite-metric quantum theory?, Czech. J. Phys. B 53, 1079 (2003). arXiv:quant-ph/0308028

[21] A. Mostafazadeh, Pseudo-Hermitian representation of quantum mechanics, Int. J. Geom. Methods Mod. Phys. 7, 1191 (2010). arXiv:0810.5643 
[22] F. K. Kunst, E. Edvardsson, J. C. Budich, and E. J. Bergholtz, Biorthogonal bulk-boundary correspondence in nonHermitian systems, Phys. Rev. Lett. 121, 026808 (2018). arXiv:1805.06492.

[23] L. Jin and Z. Song, Bulk-boundary correspondence in a non-Hermitian system in one dimension with chiral inversion symmetry, Phys. Rev. B 99, 081103 (2019). arXiv:1809.03139

[24] K. Esaki, M. Sato, K. Hasebe, and M. Kohmoto, Edge states and topological phases in non-Hermitian systems, Phys. Rev. B 84, 205128 (2011). arXiv:1106.1806.

[25] A. Ghatak and T. Das, New topological invariants in non-Hermitian systems, J. Phys.: Condens. Matter 31, 263001 (2019). arXiv:1902.07972.

[26] Q.-B. Zeng, Y.-B. Yang, and R. Lü, Topological phases in one-dimensional nonreciprocal superlattices, Phys. Rev. B 101, 125418 (2020).

[27] S. Yao, F. Song, and Z. Wang, Non-Hermitian Chern Bands, Phys. Rev. Lett. 121, 136802 (2018). arXiv:1804.04672,

[28] E. Edvardsson, F. K. Kunst, and E. J. Bergholtz, Non-Hermitian extensions of higher-order topological phases and their biorthogonal bulk-boundary correspondence, Phys. Rev. B 99, 081302 (2019). arXiv:1812.09060.

[29] V. M. Martinez Alvarez, J. E. Barrios Vargas, and L. E. F. Foa Torres, Non-Hermitian robust edge states in one dimension: Anomalous localization and eigenspace condensation at exceptional points, Phys. Rev. B 97, 121401 (2018). arXiv:1711.05235.

[30] S. Lieu, Topological phases in the non-Hermitian Su-Schrieffer-Heeger model, Phys. Rev. B 97, 045106 (2018). arXiv:1709.03788.

[31] V. M. Martinez Alvarez, J. E. Barrios Vargas, M. Berdakin, L. E. F. Foa Torres, Topological states of non-Hermitian systems, Eur. Phys. J. Spec. Top. 227, 1295 (2018). arXiv:1805.08200.

[32] A. Anderson, Canonical transformations in quantum mechanics, Ann. Phys. 232, 292 (1994). arXiv:hep-th/9305054.

[33] A. Mostafazadeh, $\mathcal{P} \mathcal{T}$-symmetric cubic anharmonic oscillator as a physical model, J. Phys. A 38, 6557 (2005); J. Phys. A 38, 8185 (2005) [erratum]. arXiv:quant-ph/0411137.

[34] R. Casalbuoni, On the quantization of systems with anticommuting variables, Nuovo Cimento A 33, 115 (1976).

[35] R. Casalbuoni, The classical mechanics for Bose-Fermi systems, Nuovo Cimento A 33, 389 (1976).

[36] F. A. Berezin and M. S. Marinov, Particle spin dynamics as the Grassmann variant of classical mechanics, Ann. Phys. 104, 336 (1977).

[37] A. Mostafazadeh, Pseudo-Hermiticity for a class of nondiagonalizable hamiltonians, J. Math. Phys. 43, 6343 (2002). arXiv:math-ph/0207009

[38] J.-P. Antoine, C. Trapani, Some remarks on quasi-Hermitian operators, J. Math. Phys. 55, 013503 (2014). arXiv:1307.5644

[39] S. Longhi, Bloch oscillations in complex crystals with $\mathcal{P} \mathcal{T}$ symmetry, Phys. Rev. Lett. 103, 123601 (2009). arXiv:1001.0981.

[40] R. P. Feynman, F. L. Vernon Jr, and R. W. Hellwarth, Geometrical representation of the Schrödinger equation for solving maser problems, J. Appl. Phys. 28, 49 (1957).

[41] Y. Ben-Aryeh, A. Mann, and I. Yaakov, Rabi oscillations in a two-level atomic system with a pseudo-Hermitian Hamiltonian, J. Phys. A 37, 12059 (2004).

[42] T. L. Gilbert, A phenomenological theory of damping in ferromagnetic materials, IEEE Trans. Magn. 40, 3443 (2004).

[43] M. Lakshmanan, The fascinating world of the Landau-Lifshitz-Gilbert equation: an overview, Philos. Trans. R. Soc. A 369, 1280 (2011). arXiv:1101.1005.

[44] E. Y. Tsymbal and I. Zutic, Handbook of Spin Transport and Magnetism (CRC Press, Boca Raton, 2016).

[45] Z. Wei, Spin-transfer-torque effect in ferromagnets and antiferromagnets, PhD Thesis, The University of Texas at Austin, Austin, TX, 2008

[46] I. N. Krivorotov, N. C. Emley, J. C. Sankey, S. I. Kiselev, D. C. Ralph, and R. A. Buhrman, Time-domain measurements of nanomagnet dynamics driven by spin-transfer torques, Science 307, 228 (2005).

[47] J. Xiao, A. Zangwill, M. D. Stiles, Macrospin models of spin transfer dynamics, Phys. Rev. B 72, 014446 (2005). arXiv:cond-mat/0504142.

[48] O. Boulle, V. Cros, J. Grollier et al., Shaped angular dependence of the spin-transfer torque and microwave generation without magnetic field. Nat. Phys. 3, 492 (2007). arXiv:0704.0370.

[49] G. Bertotti, C. Serpico, I. D. Mayergoyz, A. Magni, M. dAquino, and R. Bonin, Magnetization switching and microwave oscillations in nanomagnets driven by spinpolarized currents, Phys. Rev. Lett. 94, 127206 (2005).

[50] S. Petit, C. Baraduc, C. Thirion, U. Ebels, Y. Liu, M. Li, P. Wang, and B. Dieny, Spin-torque influence on the highfrequency magnetization fluctuations in magnetic tunnel junctions, Phys. Rev. Lett. 98, 077203 (2007).

[51] H. Y. Carr, Steady-state free precession in nuclear magnetic resonance, Phys. Rev. 112, 1693 (1958).

[52] K. Kawabata, K. Shiozaki, M. Ueda, and M. Sato, Symmetry and topology in non-hermitian physics, Phys. Rev. X 9, 041015 (2019). arXiv:1812.09133.

[53] M. C. Baldiotti and C. Molina, A second quantized approach to the Rabi problem, Braz. J. Phys. 47, 544 (2017). arXiv:1602.04828.

[54] L. Jin and Z. Song, Hermitian dynamics in a class of pseudo-Hermitian networks, Phys. Rev. A 84, 042116 (2011). arXiv:1107.4311.

[55] T. Ohashi, S. Kobayashi, and Y. Kawaguchi, Generalized Berry phase for a bosonic Bogoliubov system with exceptional points, Phys. Rev. A 101, 013625 (2020). arXiv:1904.08724.

[56] Q.-B. Zeng and Y. Xu, Winding numbers and generalized mobility edges in non-Hermitian systems, Phys. Rev. Research 2, 033052 (2020). arXiv:2002.08222. 
[57] N. Okuma, K. Kawabata, K. Shiozaki, and M. Sato, Topological origin of non-Hermitian skin effects, Phys. Rev. Lett. 124, 086801 (2020). arXiv:1910.02878.

[58] D. M. Gitman and I. V. Tyutin, Quantization of fields with constraints (Springer-Verlag, Berlin, 1991).

[59] F. A. Berezin and M. Shubin, The Schrödinger Equation (Springer, Dordrecht, 1991). 